\title{
RESPONSE OF FRACTURE NUCLEATION SITE TO WEAK MECHANICAL PULSES
}

\author{
V.S. KUKSENKO and E.E. DAMASKINSKAYA \\ Department of Strength Physics, Ioffe Physical Technical Institute Russian Academy of Sciences, St.Petersburg,
} 194021 Russia

\section{ABSTRACT}

The study is concerned with the influence of weak mechanical pulses on development of the fracture nucleation site. The macroscopic fracture of solids is considered as not an abrupt discrete event, but as a kinetic process. We assume that the fracture development in a heterogeneous material can be described in terms of the two-stage model of fracture. At the first stage, a disperse accumulation of noninteracting cracks occurs.. When the local defect concentration in some area reaches the threshold value, the prerequisite for the accelerated defect formation is set up. This area is referred to as the fracture nucleation site. Further defect accumulation provides conditions for the loss of stability of the fracture nucleation site, as a result, a larger defect is formed. Experimental data obtained for a wide fracture scale (from microcracks to earthquakes) supported the validity of the two-stage model. A study of acoustics emission (AE) due to the cyclical deformation of samples of granite, marble and sandstone was made. It has been found that AE pattern apparently depends on the stress magnitude. At a low stress, during the unloading after the first loading cycle $\mathrm{AE}$ becomes negligible and at subsequent cycles increases insignificantly. At a high stress resulting in formation of a fracture nucleation site, the unloading is not followed by a sensible drop in the AE and succeeding cycles may bring about rise of $\mathrm{AE}$ intensity. This is in agreement with the fact that at a certain stage of formation the fracture nucleation site develops independently of the stress magnitude.

\section{INTRODUCTION}

Kinetic approach to the problem of strength of solids (Zhurkov [1]) and extensive experimental data on processes of crack generation and developing (Tamuzh [2], Zhurkov [3]) suggested a model of fracture of heterogeneous materials. 


\section{PROBLEM SETTING}

We assume that the fracture development in a heterogeneous material can be described in terms of the two-stage model of fracture (Kuksenko [4]). Briefly, the key points of the model can be described as follows:

Loading of a heterogeneous material results in the crack formation. At the first stage, a disperse (diffuse) accumulation of noninteracting cracks takes place (fig.1). The sizes of these cracks are dictated by the structure scale level. The threshold value of the local defect (crack) concentration is reached in some area, the prerequisite for the accelerated defect formation is set up. This area is referred to as the fracture nucleation site. Further defect accumulation creates conditions for the loss of stability of the fracture nucleation site and, as a result, a defect is formed at the next level corresponding to the next characteristic size of the material structure.

\section{I stage}

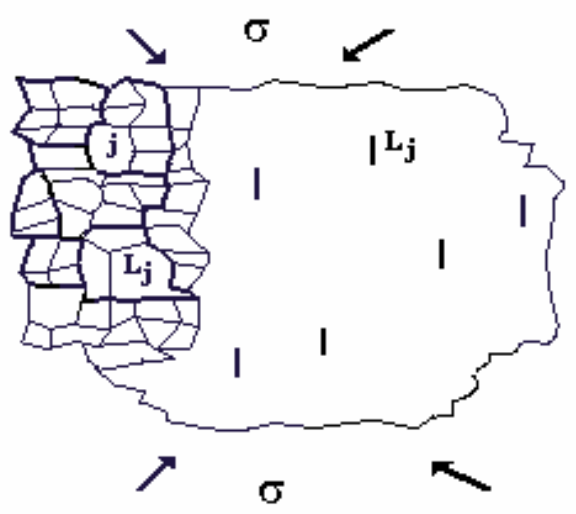

II stage

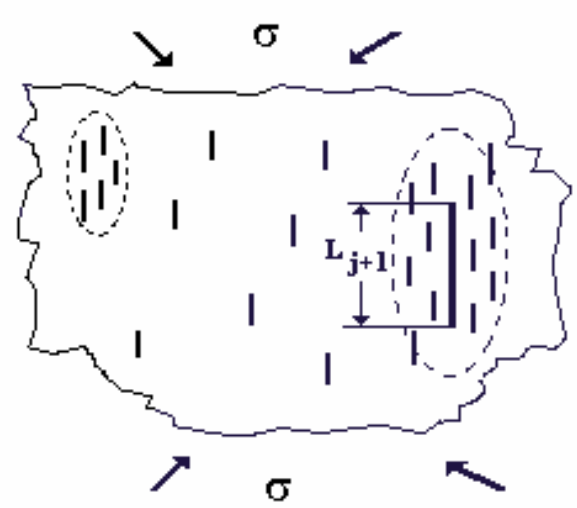

Figure 1: Scheme of the two-stage model of fracture of heterogeneous material.

The model has the advantage of validity at any structure scale level. It enables to extend the results of the laboratory sample tests to higher structure scale levels.

In our earlier works (Kuksenko [4]) we formulated the statistical criterion for the fracture nucleation site formation. It was found that a simultaneous decrease in the mean temporal $(\Delta \mathrm{t})$ intervals between chronologically successive defects and an increase in relevant variation coefficients $\left(\mathrm{V}_{\Delta \mathrm{tr}}\right)$ indicates formation of a fracture nucleation site. It was shown (Myachkin [5]) that the bulk of $\mathrm{AE}$ in rocks results from the generation of cracks. It was found as well (Kuksenko, [6]) that parameters of cracks and related AE signals are correlated. So, examination of AE gives an insight into the fracture development. 


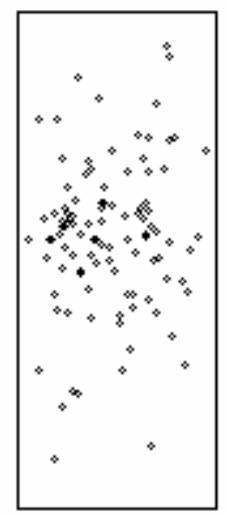

b

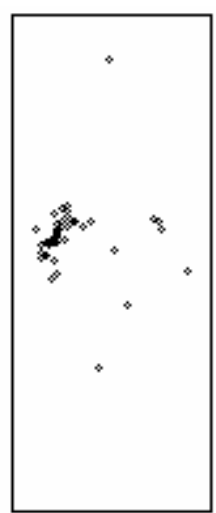

Figure 2: Spatial distribution of hypocenters of AE signals.

Fig. 2 shows the distribution of hypocenters of AE signals registered during loading of granite samples (Lockner [7], Tomilin [8]). The first (disperse) stage exhibits the uniform distribution of the AE sources through the bulk of a sample. At this stage, values of mean temporal intervals vary only slightly and variation coefficients is close to 1. From time T1, a decrease of $\Delta \mathrm{t}$ and increase of $\mathrm{V}_{\Delta \mathrm{t}}$ are observed. Localization of the defect formation begins. This is illustrated in Fig.2b, which shows distribution of hypocenters of AE sources registered after $\mathrm{T} 1$. The fracture switches to the second nucleation stage.

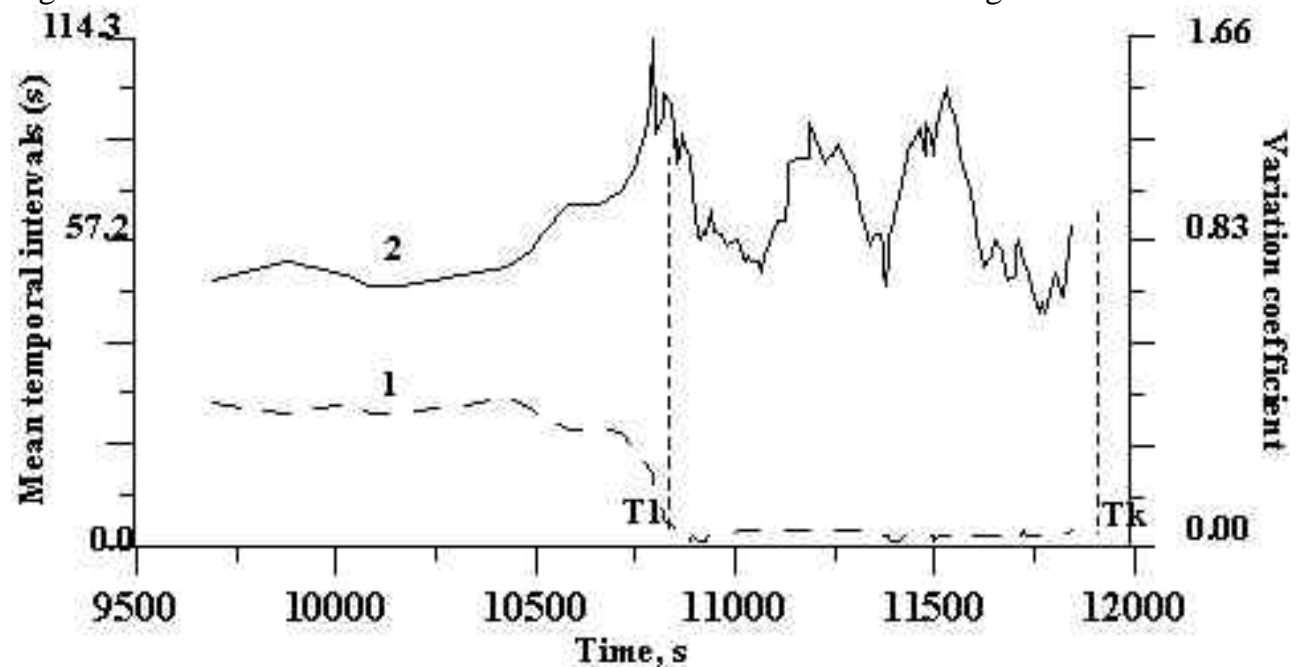

Figure 3: Variations of statistical parameters at the analysis of AE signals: mean temporal intervals (1), variation coefficient of mean temporal intervals (2). 
This method for identification of the nucleation stage onset has been extensively and successfully applied to predict the time and place of catastrophic changes in a material, however, it has some notable drawbacks. For instance, despite improvements in the AE registration techniques, the problems of exact location (deriving the coordinates of hypocenters of AE signals) and gaining adequate statistics are not conclusively solved. Besides, this method implies monitoring of the fracture development, starting with earlier stages. However, this cannot be done when testing large objects and objects that has been in use for a long time. In such cases the tests involve considerable loading resulting in not only premature aging but, perhaps, in damage to the object.

This work was aimed at investigation of behavior of the fracture nucleation site at various conditions of loading. It was expected that the regularities to be found would permit the employment of weak loading to evaluation of the extent to which a fracture nucleation site is ready for transformation to a larger defect.

\section{EXPERIMENTAL DATA AND ANALYSIS}

Granite samples shaped as a rectangular prism of the $140 \times 70 \times 70 \mathrm{~mm}^{3}$ size were subjected to loading with various magnitudes and patterns. During testing, the AE signals were registered by a fast ten-channel devise. Analysis of flow of AE signals and calculation of coordinates of the AE source hypocenters permitted us to determine with fair accuracy the time and place of arising of a fracture nucleation site capable of disintegration of a specimen. Fig. 4. shows the variation of load with time. In the same fig. changes of the AE activity are given. There were three exposures of a sample to different magnitudes of the loading.

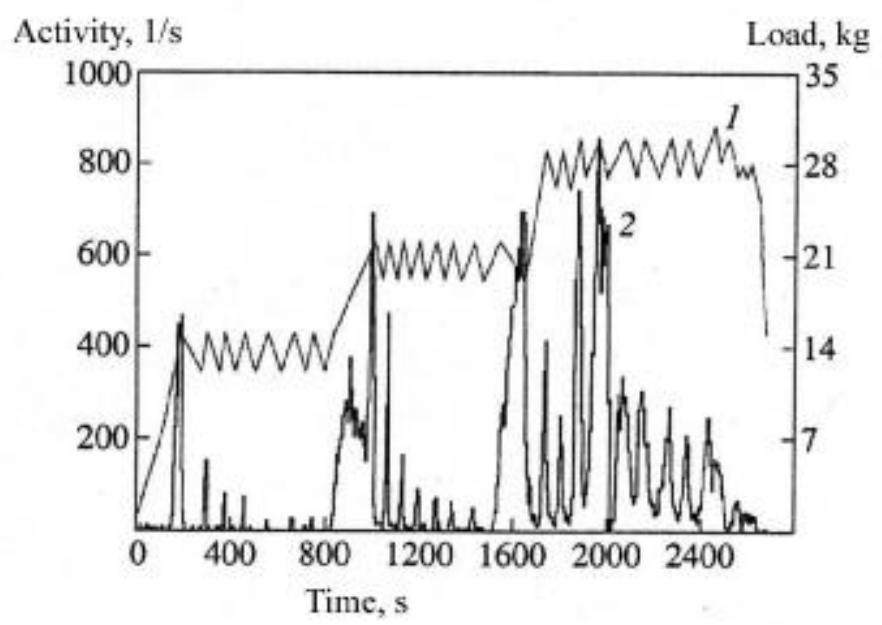

Figure 4: Temporal dependencies of load (1) and AE activity (2). 
It is seen from the plots, that in the first test $\mathrm{AE}$ intensity decreased rapidly, with increasing cycle number, down to the background noise. During the second test, the AE pattern was similar. Spatial distribution of hypocenters of AE sources implies the uniform accumulation of defects over the sample's bulk.

The third test resulted in triggering of the process to the (nucleation) stage. At that moment the static loading was interrupted and the sample was subjected to the cycled loading with the magnitude equal to that in previous tests. In this test, the AE response changed dramatically (fig. 4).

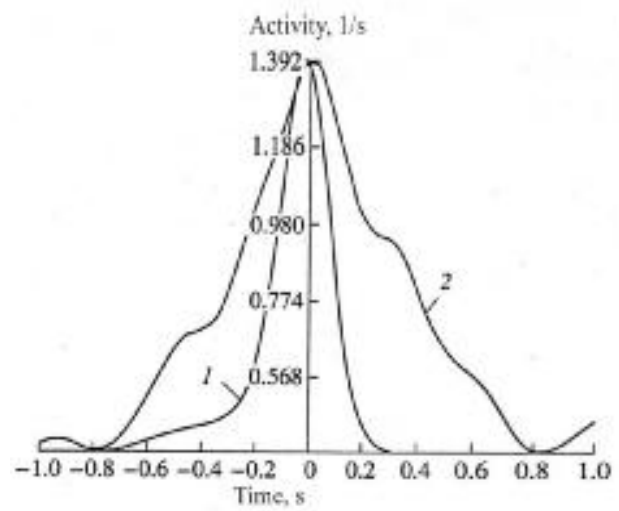

Figure 5: Temporal dependencies of normalized AE activity for disperse (1) and nucleation (2) stage.

Activity behavior was analyzed separately for increasing and decreasing load in three series of loading to find correlation between AE activity and increasing/decreasing circles of load. Normalized graphs of averaged AE activity are represented for first (curve 1) and third (curve 2) series of loading at fig.5. The negative time semiaxis corresponds to increase of load, the positive one - to decrease. The curve 1 shows that, during the first test, the acoustic activity is largely concentrated within narrow time interval close to the moment of the maximal load. Besides, the AE response is markedly asymmetrical due to rapid drop in AE intensity on unloading. The integrated activity at loading is approximately twice as large as that at unloading. The time distribution of $\mathrm{AE}$ response is considerably wider at the nucleating stage (fig.5, curve2). The absence of asymmetry in AE response is noticeable as well: ratio between integrated activities at loading and unloading is close to 1 .

\section{CONCLUSION}

The major implication of this study is that the condition of a heterogeneous material containing the mature fracture nucleation site notably differs from the initial condition. In addition, we conclude that: 
1. Arising of a fracture nucleating site in a material considerably affects the AE response of the sample to variations of load.

2. Analysis of the AE response to the cycled loading permits identification of the fracture stage (disperse or nucleation).

3. Development of a fracture nucleation site may be stimulated by a weak cycled loading.

4. Validity of the model at any structure scale level implies that methods described above can be applied to investigation of nucleation of earthquakes.

This work was supported by Russian Foundation for Basic Research (Grant N 02-0508003, N 02-05-39017, N 03-05-64831, N 04-05-65287).

\section{References}

1. Zhurkov S. N., Kinetic concept of the strength of solids, Int. J. Fracture Mech. 1, pp.311323, 1965.

2. Tamuzh, V. P., and Kuksenko, V. S., Fracture micomechanics of polymer materials (Martinus Nighoff Pub., The Hague, Boston, London), 1981.

3. Zhurkov, S. N., Kuksenko, V. S., Petrov, V. A., Principles of the kinetic approach of fracture prediction, Theoretical and Applied Fracture Mechanics, 1, pp.271-274, 1984.

4. Kuksenko V., Tomilin N., Damaskinskaya E., and Lockner D., A Two-stage Model of Fracture of Rocks // Pure Appl. Geophys. 146 (2). pp.253-263, 1996.

5. Myachkin V.I., Kostrov B.V., Sobolev G.A., Shamina O.G., Laboratory and theoretical investigations of process of Earthquake forming, Izvestiya Academy of Sciences, Earth Physics, N 10, pp .2526-2530, 1974.

6. Kuksenko V.S., Lyashkov A.I., Mirzoev K.M. at al, Correlation between crack size at loading and duration of elastic energy release, Doklady Academy of Sciences, v.264, N4, pp846-848, 1982.

7. Lockner D. A., Byerlee J. D., Kuksenko V., Ponomarev A., and Sidorin, A., Observations of quasistatic fault growth from acoustic emissions, In Fault Mechanics and Transport Properties of Rocks (ed. Evans, B. and Wong, T.-F.) (Academic Press, London) pp. 3-31, 1992.

8 .Tomilin N.G., Damaskinskaya E.E., Kuksenko V.S., Formation of a fracture focus during the deformation of heterogeneous materials (granite), Phys. Solid State, v.36, N10, pp.1649-1653, 1994. 